MAN/HEP/2007/11

SPIN-07/20, ITP-UU-07/30

\title{
Baryogenesis from the amplification of vacuum fluctuations during inflation
}

\author{
Björn Garbrecht ${ }^{1}$ and Tomislav Prokopec ${ }^{2}$ \\ ${ }^{1}$ School of Physics 85 Astronomy, The University of Manchester, \\ Oxford Road, Manchester M13 9PL, United Kingdom \\ ${ }^{2}$ Institute for Theoretical Physics (ITF) 8 Spinoza Institute, Utrecht University, \\ Leuvenlaan 4, Postbus 80.195, 3508 TD Utrecht, The Netherlands
}

\begin{abstract}
We propose that the baryon asymmetry of the Universe may originate from the amplification of quantum fluctuations of a light complex scalar field during inflation. $\mathrm{CP}$-violation is sourced by complex mass terms, which are smaller than the Hubble rate, as well as non-standard kinetic terms. We find that, when assuming 60 e-folds of inflation, an asymmetry in accordance with observation can result for models where the energy scale of inflation is of the order of $10^{16} \mathrm{GeV}$. Lower scales may be achieved when assuming substantially larger amounts of e-folds.
\end{abstract}

\section{Introduction}

It was pointed out by Sakharov that the matter-antimatter asymmetry of the Universe does not need to be put in as an initial condition set up at the Big Bang, but can be explained within models of particle physics, even if the initial asymmetry is assumed to be zero [1]. This is an important and celebrated observation, and in fact the failure of the Standard Model to explain the baryon number of the Universe (BAU) is one of the chief motivations for considering its extensions. The inflationary paradigm further augments the urge for an explanation of the BAU from particle physics, since due to the increase of its volume by at least a factor of about $10^{70}$, the Universe is essentially void of particles at the end of inflation. Any possible pre-existing asymmetry would have been diluted away.

Loopholes to this argument appear to exist. Recently, a model within an extended, CPviolating Brans-Dicke model and $\mathrm{CP}$ violation in the kinetic term has been proposed [2]. The authors have shown that an initial classical lump of scalar charge can be amplified and stretched to large scales during inflation. After inflation the charge is then converted into a baryon asymmetry.

While Ref. 2] deals with amplification of classical charge density, here we show that, analogously to cosmological perturbations, a similar scalar field model can be used to amplify quantum charge density fluctuations during inflation. In this paper, we point out that indeed, a negative mass term is not necessary to generate a sufficient BAU. For a complex scalar field with CP-violating masses and kinetic terms, the growth of the modes 
due to the expanding background can lead to a sufficient BAU, provided the physical mass eigenvalues for the scalar degrees of freedom do not exceed the Hubble expansion rate. The mechanism of particle production is the same as for the generation of density perturbations from inflation, supplemented however with CP-violation.

For simplicity we perform our analysis in de Sitter space. We show that charged quantum fluctuations are generically amplified to a charge density that is on super-Hubble wavelengths independent on scale (scale invariant spectrum). Moreover, when integrated out, at the end of inflation one obtains a scalar charge density $q_{\phi}$ of the form (see Eq. (4.15)),

$$
q_{\phi}=\left|\omega \mu^{2}\right| H \sin \theta_{\mathrm{CP}}\left[-\frac{3}{2 \pi^{2}} \frac{H^{2} \dot{H}}{m^{4}-|\mu|^{4}}+\frac{1}{8 \pi^{2}} \frac{1}{\varrho^{2}-|\omega|^{2}}\right]
$$

where $\mu$ and $m$ are the $\mathrm{CP}$ odd and even masses, $\rho$ and $\omega$ are the (dimensionless) couplings of the CP even and odd kinetic terms, respectively ( $c f$. Eq. (2.1)) and $\dot{H}=d H / d t$ is the rate of change of the Hubble parameter $H$. As expected, according to the result (1.1) the charge vanishes either when $\mathrm{CP}$ violation vanishes (which is the case when any of the parameters $\omega, \mu$ or $\theta_{\mathrm{CP}}$ vanishes) or when $H$ vanishes (because the Universe's expansion is the source for the amplification of charged vacuum fluctuations). The result (1.1) allows us to establish a relation between inflationary observables and baryogenesis, which we discuss in some detail in section 6 .

Conserved currents usually give a unique prescription to define charges. In turn, when there is no exactly conserved current, the definition of a charge is in general ambiguous. In practice, this is most often not a problem due to the existence of hierarchies in physical models. For example, consider standard leptogenesis. Within the Standard Model, the baryon minus lepton number $B-L$ is conserved, but when Majorana neutrinos are added, it is not. Nonetheless, since the Majorana neutrinos are usually either chosen to be much heavier than Standard Model particles or very weakly coupled to these, it is of course reasonable to define $B-L$ from the conserved global charge in the Standard Model, even though it is violated in the full theory.

In section 2, we take the view that the scalar field, which carries the initial charge asymmetry, decays into much lighter fermions, which carry $B-L$ charge. This leads us to a current which is conserved in the fermionic sector but violated for the scalar field, analogous to the above example. In section 3 we quantize the scalar field and make use of this to calculate the current at late times during inflation in section 4. Possible particle physics models as realizations of our mechanism are sketched in section 5 , and a quantitative discussion of the BAU including the relation to the scale of inflation is the content of 6 .

\section{CP violation and charged current}

In this section, we introduce a scalar lagrangian which is endowed with CP-violation, such that a charge asymmetry can result from vacuum fluctuations. In order to obtain a meaningful notion of a charge number, we also define the coupling of the scalar to fermions. This leads us to a current that is approximately conserved when the scalar field decays into the fermions. The calculation of this current is the subject of the subsequent sections. 
The most general quadratic lagrangian for a single complex scalar field, which is minimally coupled to curvature, is given by

$$
\mathcal{L}_{\phi}=\sqrt{-g}\left[\varrho g^{\mu \nu}\left(\partial_{\mu} \phi\right) \partial_{\nu} \phi^{*}+\frac{\omega}{2} g^{\mu \nu} \partial_{\mu} \phi \partial_{\nu} \phi+\frac{\omega^{*}}{2} g^{\mu \nu} \partial_{\mu} \phi^{*} \partial_{\nu} \phi^{*}-m^{2} \phi \phi^{*}-\frac{\mu^{2}}{2} \phi^{2}-\frac{\mu^{* 2}}{2} \phi^{* 2}\right] \text {. }
$$

We assume an inflationary background and use conformal coordinates, such that the metric is $g_{\mu \nu}=a^{2} \eta_{\mu \nu}$ with the scale factor

$$
a=-\frac{1}{H \eta}
$$

where $\eta<0$ denotes the conformal time and $H$ the Hubble rate. The deviation of inflationary expansion from an exact de Sitter phase can be incorporated by allowing for a weak time dependence of $H$. A rigorous treatment of the charge production in quasi de Sitter spaces can be performed by making use of the quasi de Sitter scalar propagator [3]. We furthermore introduce a Dirac fermion coupling to the scalar field as

$$
\mathcal{L}_{\psi}=\sqrt{-g}\left[\bar{\psi}_{L} \mathrm{i} \not \partial \psi_{L}+\bar{\psi}_{R} \mathrm{i} \not \partial \psi_{R}-f\left(\bar{\psi}_{R} \phi \psi_{L}+\text { h.c. }\right)\right]
$$

where $f$ is a real Yukawa coupling. The full lagrangian density is then given by

$$
\mathcal{L}=\mathcal{L}_{\phi}+\mathcal{L}_{\psi}
$$

It is of course possible to redefine $\phi$ in such a way that the lagrangian (2.1) has a canonical kinetic term. In section 3, we perform such a redefinition explicitly. In that case, the Yukawa couplings in (2.3) get more complicated. For the definition of a current, which is conserved during the decays to fermions, we however find the present field definition more useful.

Starting point for the definition of the current is a $\mathrm{U}(1)$-charge, under which

$$
\begin{aligned}
\psi_{R} & \rightarrow \mathrm{e}^{-\mathrm{i} \beta} \psi_{R}, \\
\psi_{L} & \rightarrow \mathrm{e}^{-\mathrm{i}(\alpha+\beta)} \psi_{L}, \\
\phi & \rightarrow \mathrm{e}^{\mathrm{i} \alpha} \phi .
\end{aligned}
$$

These transformation properties are chosen in such a way that the Yukawa terms are singlets, i.e. during the decay of a $\phi$-particle to a pair of fermions, the $\mathrm{U}(1)$-charge remains conserved. Up to a normalization, this requirement defines the above symmetry and current in a unique way. If after the complete decay of $\phi$ a certain charge is stored in the $\psi_{L}$, we are interested in how to express this charge as a function in terms of $\phi$ before the decay.

Next, we recall some facts about symmetry currents. Let $\mathcal{L}_{\varphi}$ be a lagrangian and $\varphi$ a scalar field. Performing an infinitesimal transformation $\varphi \rightarrow \varphi+\varepsilon \Delta \varphi$ has the effect $\mathcal{L}_{\varphi} \rightarrow \mathcal{L}_{\varphi}+\varepsilon \Delta \mathcal{L}_{\varphi}$ on the lagrangian, where

$$
\Delta \mathcal{L}_{\varphi}=\frac{\partial \mathcal{L}_{\varphi}}{\partial \varphi} \Delta \varphi+\left(\frac{\partial \mathcal{L}_{\varphi}}{\partial\left(\partial_{\mu} \varphi\right)}\right) \partial_{\mu} \Delta \varphi=\partial_{\mu}\left(\frac{\partial \mathcal{L}_{\varphi}}{\partial\left(\partial_{\mu} \varphi\right)} \Delta \varphi\right)+\left[\frac{\partial \mathcal{L}_{\varphi}}{\partial \varphi}-\partial_{\mu} \frac{\partial \mathcal{L}_{\varphi}}{\partial\left(\partial_{\mu} \varphi\right)}\right] \Delta \varphi
$$

and the last term vanishes by virtue of the Euler-Lagrange equation. If $\Delta \mathcal{L}_{\varphi}$ is zero or it is possible to write it as a total divergence $\Delta \mathcal{L}_{\varphi}=\partial_{\mu} \mathcal{J}^{\mu}$, we can define the conserved current

$$
j_{\varphi}^{\mu}=\frac{\partial \mathcal{L}_{\varphi}}{\partial\left(\partial_{\mu} \varphi\right)} \Delta \varphi-\mathcal{J}^{\mu},
$$


which satisfies $\partial_{\mu} j_{\varphi}^{\mu}=0$.

For the symmetry (2.5), we use

$$
j_{\phi}^{\mu}=\frac{\partial \mathcal{L}_{\phi}}{\partial\left(\partial_{\mu} \phi\right)} \Delta \phi
$$

where $\Delta \phi=\mathrm{i} \phi$ (taking in (2.5) an infinitesimal $\alpha=\varepsilon$ ), such that we find

$$
j_{\phi}^{\mu}=\sqrt{g} g^{\mu \nu}\left\{\mathrm{i} \varrho\left[\left(\partial_{\nu} \phi^{*}\right) \phi-\phi^{*}\left(\partial_{\nu} \phi\right)\right]+\left[\mathrm{i} \omega\left(\partial_{\nu} \phi\right) \phi+\text { c.c. }\right]\right\} .
$$

In this case however, $\Delta \mathcal{L}$ is non-zero and can neither be expressed in terms of a total divergence, which means that the symmetry (2.5) is violated. Explicitly,

$$
\Delta \mathcal{L}=\sqrt{-g}\left\{\mathrm{i} \omega g^{\mu \nu} \partial_{\mu} \phi \partial_{\nu} \phi-\mathrm{i} \mu^{2} \phi^{2}+\text { h.c. }\right\} .
$$

This terms originates from $\mathcal{L}_{\phi}$ only and has no contribution from $\mathcal{L}_{\psi}$, according to our requirement that the decay of the scalar to fermions and the fermionic sector itself should respect the current. Of course, the total current $j_{\phi, \psi}^{\mu}=j_{\phi}^{\mu}+j_{\psi}^{\mu}$ also has the fermionic contribution

$$
j_{\psi}^{\mu}=\sqrt{-g} \bar{\psi}_{L} \Gamma^{\mu} \psi_{L}
$$

where $\Gamma^{\mu}$ denotes the $\gamma$-matrices contracted with the vierbein linking curved space with a local Minkowskian coordinate system. For a conformal background $g_{\mu \nu}=a^{2} \eta_{\mu \nu}$, as assumed in this paper, we have $\Gamma^{\mu}=(1 / a) \gamma^{\mu}$

The U(1)-violation during inflation as expressed by (2.10) can be the source for successful baryogenesis. On the other hand, we have to require that after inflation, the once generated charge is not erased. It is therefore important that the field $\phi$ does not thermalize and that it quickly decays into the fermions. Under the conditions

$$
f^{2} m /(8 \pi) \gtrsim \mu \text { and } \varrho \gtrsim \omega
$$

the decay rate into fermions is

$$
\Gamma_{\phi \rightarrow \bar{\psi} \psi} \approx f^{2} \sqrt{m^{2} / \varrho} /(8 \pi),
$$

whereas the rate of $\mathrm{U}(1)$-violation is of the order of

$$
\partial_{0} j^{0} /\left(j^{0}\right)=O(\mu / \sqrt{\varrho}, m \sqrt{\omega / \varrho}),
$$

such that the requirement

$$
\partial_{0} j^{0} /\left(j^{0}\right) \lesssim \Gamma_{\phi \rightarrow \bar{\psi} \psi}
$$

is satisfied.

\section{Real field decomposition}

In this section, we quantize the scalar field $\phi$ as defined by the lagrangian (2.1). In order to do so, we decompose $\phi$ into canonically normalized real degrees of freedom mixing through a real symmetric mass-square matrix $M^{2}$. 
For the complex scalar field $\phi$, it is always possible to go to the real field basis

$$
\phi=\alpha \phi_{+}+i \beta \phi_{-}, \quad \phi^{\dagger}=\alpha^{*} \phi_{+}-i \beta^{*} \phi_{-}, \quad\left(\phi_{+}=\frac{\beta^{*} \phi+\beta \phi^{\dagger}}{2 \Re\left[\alpha \beta^{*}\right]}, \phi_{-}=\frac{\alpha^{*} \phi-\alpha \phi^{\dagger}}{2 i \Re\left[\alpha \beta^{*}\right]}\right) .
$$

Inserting this into the lagrangian (2.1) and demanding that the kinetic terms are canonically normalized we obtain

$$
\begin{aligned}
-i \varrho\left(\alpha \beta^{*}-\alpha^{*} \beta\right)+i \omega \alpha \beta-i(\omega \alpha \beta)^{*} & =0, \\
\varrho|\alpha|^{2}+\frac{1}{2} \omega \alpha^{2}+\frac{1}{2}\left(\omega \alpha^{2}\right)^{*} & =\frac{1}{2}, \\
\varrho|\beta|^{2}-\frac{1}{2} \omega \beta^{2}-\frac{1}{2}\left(\omega \beta^{2}\right)^{*} & =\frac{1}{2} .
\end{aligned}
$$

With these substitutions the lagrangian (2.1) becomes

$$
\mathcal{L}_{\phi}=\sqrt{-g}\left[\frac{1}{2} \sum_{ \pm} g^{\mu \nu}\left(\partial_{\mu} \phi_{ \pm}\right) \partial_{\nu} \phi_{ \pm}-\frac{1}{2} m_{+}^{2} \phi_{+}^{2}-\frac{1}{2} m_{-}^{2} \phi_{-}^{2}-m_{+-}^{2} \phi_{+} \phi_{-}\right]
$$

where the mass-squares are

$$
\begin{aligned}
m_{+}^{2} & =2|\alpha|^{2}\left[m^{2}+|\mu|^{2} \cos \left(2 \theta_{\mu}+2 \theta_{\alpha}\right)\right] \\
m_{-}^{2} & =2|\beta|^{2}\left[m^{2}-|\mu|^{2} \cos \left(2 \theta_{\mu}+2 \theta_{\beta}\right)\right] \\
m_{+-}^{2} & =2|\alpha||\beta|\left[m^{2} \sin \left(\theta_{\alpha}-\theta_{\beta}\right)-|\mu|^{2} \sin \left(2 \theta_{\mu}+\theta_{\alpha}+\theta_{\beta}\right)\right]
\end{aligned}
$$

and where we defined

$$
\omega=|\omega| \mathrm{e}^{i \theta_{\omega}}, \quad \alpha=|\alpha| \mathrm{e}^{i \theta_{\alpha}}, \quad \beta=|\beta| \mathrm{e}^{i \theta_{\beta}} .
$$

Eqs. (3.2 3.4) represent two real and one imaginary condition for two complex numbers, which means that $\alpha$ and $\beta$ are not completely specified. The conditions (3.2 3.4) can be recast as

$$
\begin{aligned}
\varrho \sin \left(\theta_{-}\right)-|\omega| \sin \left(\theta_{+}\right) & =0 \\
\varrho|\alpha|^{2}+\frac{1}{2}|\omega||\alpha|^{2} \cos \left(\theta_{+}+\theta_{-}\right) & =\frac{1}{2}, \\
\varrho|\beta|^{2}+\frac{1}{2}|\omega||\beta|^{2} \cos \left(\theta_{+}-\theta_{-}\right) & =\frac{1}{2},
\end{aligned}
$$

where we introduced the angles

$$
\theta_{+}=\theta_{\omega}+\theta_{\alpha}+\theta_{\beta}, \quad \theta_{-}=\theta_{\alpha}-\theta_{\beta} .
$$

¿From the first equation (3.10) we get

$$
\sin \left(\theta_{-}\right)=\frac{|\omega|}{\varrho} \sin \left(\theta_{+}\right)
$$


while the latter two equations 3.113 .12 imply

$$
\begin{aligned}
\cos \left(\theta_{+}\right) \cos \left(\theta_{-}\right) & =\frac{1}{4|\omega|}\left(\frac{1}{|\alpha|^{2}}-\frac{1}{|\beta|^{2}}\right), \\
\sin \left(\theta_{+}\right) \sin \left(\theta_{-}\right) & =\frac{\varrho}{|\omega|}-\frac{1}{4|\omega|}\left(\frac{1}{|\alpha|^{2}}+\frac{1}{|\beta|^{2}}\right) .
\end{aligned}
$$

From (3.16) and (3.14) we then find

$$
\sin ^{2}\left(\theta_{-}\right)=1-\frac{1}{4 \varrho}\left(\frac{1}{|\alpha|^{2}}+\frac{1}{|\beta|^{2}}\right) .
$$

Finally, Eq. (3.15) can be solved to give the following relation between $|\alpha|$ and $|\beta|$,

$$
|\alpha|^{2}+|\beta|^{2}=\frac{\varrho}{\varrho^{2}-|\omega|^{2}} .
$$

As promised the solution is not unique. Indeed, there is a one parameter family of solutions. A particularly convenient choice is

$$
|\alpha|^{2}=|\beta|^{2}=\frac{1}{2} \frac{\varrho}{\varrho^{2}-|\omega|^{2}},
$$

for which we then have

$$
\sin \left(\theta_{+}\right)=1, \quad \cos \left(\theta_{+}\right)=0, \quad \theta_{+}=\frac{\pi}{2}, \quad \sin \left(\theta_{-}\right)=\frac{|\omega|}{\varrho}, \quad \cos \left(\theta_{-}\right)=\sqrt{1-\frac{|\omega|^{2}}{\varrho^{2}}} .
$$

With this choice the mass-squares (3.6 3.8) become

$$
\begin{aligned}
m_{+}^{2} & =\frac{\varrho}{\varrho^{2}-|\omega|^{2}}\left[m^{2}+|\mu|^{2} \cos \left(\theta_{\mathrm{CP}}+\theta_{+}+\theta_{-}\right)\right] \\
& =\frac{\varrho}{\varrho^{2}-|\omega|^{2}}\left[m^{2}-|\mu|^{2}\left(\frac{|\omega|}{\varrho} \cos \left(\theta_{\mathrm{CP}}\right)+\sqrt{\left.\left.1-\frac{|\omega|^{2}}{\varrho^{2}} \sin \left(\theta_{\mathrm{CP}}\right)\right)\right]}\right.\right. \\
m_{-}^{2} & =\frac{\varrho}{\varrho^{2}-|\omega|^{2}}\left[m^{2}-|\mu|^{2} \cos \left(\theta_{\mathrm{CP}}+\theta_{+}-\theta_{-}\right)\right] \\
& =\frac{\varrho}{\varrho^{2}-|\omega|^{2}}\left[m^{2}-|\mu|^{2}\left(\frac{|\omega|}{\varrho} \cos \left(\theta_{\mathrm{CP}}\right)-\sqrt{1-\frac{|\omega|^{2}}{\varrho^{2}}} \sin \left(\theta_{\mathrm{CP}}\right)\right)\right], \\
m_{+-}^{2} & =\frac{\varrho}{\varrho^{2}-|\omega|^{2}}\left[m^{2} \sin \left(\theta_{-}\right)-|\mu|^{2} \sin \left(\theta_{\mathrm{CP}}+\theta_{+}\right)\right] \\
& =\frac{\varrho}{\varrho^{2}-|\omega|^{2}}\left[m^{2} \frac{|\omega|}{\varrho}-|\mu|^{2} \cos \left(\theta_{\mathrm{CP}}\right)\right]
\end{aligned}
$$

where we defined the CP angle

$$
\theta_{\mathrm{CP}}=2 \theta_{\mu}-\theta_{\omega} .
$$

The lagrangian (3.5) implies the following field equations:

$$
\begin{aligned}
& \frac{1}{a^{2}}\left(a^{2} \phi_{+}^{\prime}\right)^{\prime}+\left(-\partial_{i}^{2}+a^{2} m_{+}^{2}\right) \phi_{+}+a^{2} m_{+-}^{2} \phi_{-}=0, \\
& \frac{1}{a^{2}}\left(a^{2} \phi_{-}^{\prime}\right)^{\prime}+\left(-\partial_{i}^{2}+a^{2} m_{-}^{2}\right) \phi_{-}+a^{2} m_{+-}^{2} \phi_{+}=0,
\end{aligned}
$$


where a prime denotes a derivative with respect to $\eta$. When introducing the mass-square matrix

$$
M^{2} \equiv\left(\begin{array}{cc}
m_{+}^{2} & m_{+-}^{2} \\
m_{+-}^{2} & m_{-}^{2}
\end{array}\right)
$$

these can be compactly written as

$$
\frac{1}{a^{2}}\left(a^{2} \Phi^{\prime}\right)^{\prime}+\left(-\partial_{i}^{2}+a^{2} M^{2}\right) \Phi=0,
$$

where we defined

$$
\Phi=\left(\begin{array}{c}
\phi_{+} \\
\phi_{-}
\end{array}\right)
$$

The conformally rescaled form of Eq. (3.27) is

$$
\left(\partial_{\eta}^{2}-\partial_{i}^{2}+a^{2} M^{2}-\frac{a^{\prime \prime}}{a}\right)(a \Phi)=0 \text {. }
$$

We now perform the canonical quantization by imposing the commutator

$$
\left[\hat{\phi}_{a}(\vec{x}, \eta), \hat{\pi}_{b}\left(\vec{x}^{\prime}, \eta\right)\right]=\mathrm{i} \delta_{a b} \delta^{3}\left(\vec{x}-\vec{x}^{\prime}\right) \quad(a, b= \pm, \hbar=1)
$$

and the canonical momentum equals

$$
\hat{\pi}_{a}=\frac{\partial \mathcal{L}_{\phi}}{\partial \partial_{\eta} \phi_{a}}=a^{2} \hat{\phi}_{a}^{\prime}, \quad(a= \pm)
$$

or equivalently

$$
\hat{\Pi}=a^{2} \hat{\Phi}^{\prime}
$$

with

$$
\hat{\Phi}=\left(\begin{array}{c}
\hat{\phi}_{+} \\
\hat{\phi}_{-}
\end{array}\right), \quad \hat{\Pi}=\left(\begin{array}{c}
\hat{\pi}_{+} \\
\hat{\pi}_{-}
\end{array}\right) .
$$

Since the system (3.25) we want to solve is linear and the mass matrix (3.26) is real symmetric, the field can be expressed in terms of the annihilation and creation operators as

$$
\phi_{a}(x)=\sum_{b= \pm} \int \frac{d^{3} k}{(2 \pi)^{3}} \mathrm{e}^{\mathrm{i} \vec{k} \cdot \vec{x}}\left[\phi_{k, a b}(\eta) \hat{a}_{\vec{k}, b}+\phi_{k, a b}^{*}(\eta) \hat{a}_{-\vec{k}, b}^{\dagger}\right], \quad(a, b= \pm, k=\|\vec{k}\|),
$$

or in a matrix form,

$$
\Phi(x)=\int \frac{d^{3} k}{(2 \pi)^{3}} \mathrm{e}^{\mathrm{i} \vec{k} \cdot \vec{x}}\left[\Phi_{k}(\eta) \cdot \hat{A}_{\vec{k}}+\Phi_{k}^{*}(\eta) \cdot \hat{A}_{-\vec{k}}^{\dagger}\right],
$$

where

$$
\Phi_{k}=\left(\begin{array}{cc}
\phi_{k,++} & \phi_{k,+-} \\
\phi_{k,-+} & \phi_{k,--}
\end{array}\right), \quad \hat{A}_{\vec{k}}=\left(\begin{array}{c}
\hat{a}_{\vec{k},+} \\
\hat{a}_{\vec{k},-}
\end{array}\right) .
$$

Note that the symmetry of $M^{2}$ also implies that if $\Phi_{k}(\eta)$ and $\Phi_{k}^{\prime}(\eta)$ are symmetric for a particular $\eta$, they are symmetric at all times. In particular, we impose the symmetry of 
$\Phi_{k}$ and $\Phi_{k}^{\prime}$ as initial conditions and, in the following, make frequently use of the symmetry property $\Phi_{k}^{*}(\eta)=\Phi_{k}^{\dagger}(\eta)$.

Upon inserting (3.35) into (3.29) we get for the mode equation

$$
\left(\partial_{\eta}^{2}+k^{2}+a^{2} M^{2}-\frac{a^{\prime \prime}}{a}\right)\left(a \Phi_{k}(\eta)\right)=0 .
$$

This equation can be solved in de Sitter space with the scale factor (2.2). The two fundamental solutions can be written as

$$
\Phi_{k}(\eta)=\frac{1}{a} \sqrt{-\frac{\pi \eta}{4}} \mathrm{e}^{\mathrm{i}(\pi / 2)(\nu-1 / 2)} H_{\nu}^{(1)}(-k \eta), \quad \Phi_{k}^{\dagger}(\eta) \quad\left(\nu^{2}=\frac{9}{4}-\frac{M^{2}}{H^{2}}\right) .
$$

This is a matrix generalization of the standard Bunch-Davies vacuum solution for de Sitter space [4]. Since the mass matrix is time-independent, the Hankel functions in (3.38) can be ascribed a precise meaning. Also, since there is only one matrix in (3.38), the ordering of terms in Eq. (3.38) is irrelevant. The general mode functions are then a linear combination of the fundamental solutions (3.38),

$$
\Xi=\gamma_{1} \cdot \Phi_{k}(\eta)+\gamma_{2} \cdot \Phi_{k}(\eta)^{\dagger}, \quad \Xi^{\dagger}=\Phi_{k}^{\dagger}(\eta) \cdot \gamma_{1}+\Phi_{k}(\eta) \cdot \gamma_{2},
$$

where $\gamma_{1}$ and $\gamma_{2}$ are constant $2 \times 2$ matrices. Since $\nu$ is a constant matrix, it is easily checked that the Wronskian of the fundamental solutions (3.38) is the simple generalization of the single field case,

$$
W\left[\Phi_{k}(\eta), \Phi_{k}^{\dagger}(\eta)\right]=\Phi_{k}(\eta) \cdot \Phi_{k}^{\dagger^{\prime}}(\eta)-\Phi_{k}^{\prime}(\eta) \cdot \Phi_{k}^{\dagger}(\eta)=\frac{\mathrm{i}}{a^{2}}
$$

Requiring that the Wronskian of the general mode function (3.39) be unchanged leads to the following condition on $\gamma_{1}$ and $\gamma_{2}$,

$$
\gamma_{1} \cdot \gamma_{1}^{\dagger}-\gamma_{2} \cdot \gamma_{2}^{\dagger}=\mathbf{1}
$$

In this work we shall take the initial vacuum state corresponding to zero particles as $\eta \rightarrow-\infty$, in which case we have $\gamma_{1}=\operatorname{diag}(1,1)$ and $\gamma_{2}=\operatorname{diag}(0,0)$.

\section{Scalar current}

In this section, we apply the scalar field decomposition and quantization to calculate the scalar charge generated during inflation. The comoving scalar current (2.9) in conformal coordinates is

$$
{\hat{j_{\phi}}}^{\mu}=a^{4}\left\{\mathrm{i} \varrho\left[\left(\partial^{\mu} \hat{\phi}^{\dagger}\right) \hat{\phi}-\hat{\phi}^{\dagger}\left(\partial^{\mu} \hat{\phi}\right)\right]+\mathrm{i} \omega\left(\partial^{\mu} \hat{\phi}\right) \hat{\phi}-\mathrm{i} \omega^{*}\left(\partial^{\mu} \hat{\phi}^{\dagger}\right) \hat{\phi}^{\dagger}\right\}
$$

which, when expressed in terms of the real fields, becomes

$$
\begin{aligned}
{\hat{j_{\phi}}}^{\mu} & =a^{4}\left\{\left[\mathrm{i} \omega \alpha^{2}-\mathrm{i}\left(\omega \alpha^{2}\right)^{*}\right] \hat{\phi}_{+} \partial^{\mu} \hat{\phi}_{+}-\left[\mathrm{i} \omega \beta^{2}-\mathrm{i}\left(\omega \beta^{2}\right)^{*}\right] \hat{\phi}_{-}\left(\partial^{\mu} \hat{\phi}_{-}\right)\right. \\
& \left.+\left[-\varrho\left(\alpha \beta^{*}+\alpha^{*} \beta\right)-\omega \alpha \beta-(\omega \alpha \beta)^{*}\right] \hat{\phi}_{-} \partial^{\mu} \hat{\phi}_{+}+\left[\varrho\left(\alpha \beta^{*}+\alpha^{*} \beta\right)-\omega \alpha \beta-(\omega \alpha \beta)^{*}\right] \hat{\phi}_{+} \partial^{\mu} \hat{\phi}_{-}\right\} .
\end{aligned}
$$


With the choice (3.20) this reduces to

$$
\hat{j}_{\phi}^{\mu}=a^{4}\left\{-\frac{|\omega|}{\sqrt{\varrho^{2}-|\omega|^{2}}}\left[\hat{\phi}_{+} \partial^{\mu} \hat{\phi}_{+}-\hat{\phi}_{-} \partial^{\mu} \hat{\phi}_{-}\right]+\frac{\varrho}{\sqrt{\varrho^{2}-|\omega|^{2}}}\left[\hat{\phi}_{+} \partial^{\mu} \hat{\phi}_{-}-\hat{\phi}_{-} \partial^{\mu} \hat{\phi}_{+}\right]\right\} \text {. }
$$

We now use the solution (3.35) and (3.38) to calculate the expectation value of the current (4.3) in the vacuum $\hat{a}_{\vec{k}, b}|0\rangle=0$. Noting $\partial_{\mu}=\left(\partial_{\eta}, \partial / \partial x_{i}\right)$, we find

$$
\begin{aligned}
j_{\phi}{ }^{\mu} \equiv & \left\langle 0\left|\hat{j}_{\phi}^{\mu}\right| 0\right\rangle \\
= & -a^{2} \frac{|\omega|}{\sqrt{\varrho^{2}-|\omega|^{2}}} \int \frac{d^{3} k}{(2 \pi)^{3}}\left[\left(\Phi_{k}(\eta) \cdot\left(\partial_{\eta}, i k_{i}\right) \Phi_{k}^{\dagger}(\eta)\right)_{++}-\left(\Phi_{k}(\eta) \cdot\left(\partial_{\eta}, i k_{i}\right) \Phi_{k}^{\dagger}(\eta)\right)_{--}\right] \\
& +a^{2} \frac{\varrho}{\sqrt{\varrho^{2}-|\omega|^{2}}} \int \frac{d^{3} k}{(2 \pi)^{3}}\left[\left(\Phi_{k}(\eta) \cdot\left(\partial_{\eta}, i k_{i}\right) \Phi_{k}^{\dagger}(\eta)\right)_{+-}-\left(\Phi_{k}(\eta) \cdot\left(\partial_{\eta}, i k_{i}\right) \Phi_{k}^{\dagger}(\eta)\right)_{-+}\right],
\end{aligned}
$$

where we made use of

$$
\left\langle 0\left|\hat{\Phi} \cdot \partial_{\mu} \hat{\Phi}\right| 0\right\rangle=\int \frac{d^{3} k}{(2 \pi)^{3}} \Phi_{k}(\eta) \cdot \partial_{\mu} \Phi_{k}^{\dagger}(\eta)
$$

The spatial part of the current (4.4) vanishes when integrated over the momentum, so that we need to calculate only the 0th component. Moreover, since the mass matrix is symmetric, so is $\Phi$, and hence $\Phi^{\dagger}=\Phi^{*}$. This allows one to rewrite the time component of Eq. (4.5) as

$$
\Phi_{k}(\eta) \cdot \partial_{\eta} \Phi_{k}^{\dagger}(\eta)=\frac{i}{2 a^{2}}+\frac{1}{2} \partial_{\eta}\left\{\Phi_{k}(\eta) \cdot \Phi_{k}^{*}(\eta)\right\}
$$

where we made use of the Wronskian (3.40).

To get the physical charge density $q_{\phi}$, we recall that $j_{\phi}^{0}$ corresponds to the comoving charge. Therefore, one ought to multiply (4.4) by $1 / a^{3}$ :

$$
\begin{aligned}
q_{\phi}= & \frac{j_{\phi}^{0}}{a^{3}}=-\frac{1}{a} \frac{|\omega|}{\sqrt{\varrho^{2}-|\omega|^{2}}} \int \frac{d^{3} k}{(2 \pi)^{3}}\left[\left(\Phi_{k}(\eta) \cdot \partial_{\eta} \Phi_{k}^{\dagger}(\eta)\right)_{++}-\left(\Phi_{k}(\eta) \cdot \partial_{\eta} \Phi_{k}^{\dagger}(\eta)\right)_{--}\right] \\
& +\frac{1}{a} \frac{\varrho}{\sqrt{\varrho^{2}-|\omega|^{2}}} \int \frac{d^{3} k}{(2 \pi)^{3}}\left[\left(\Phi_{k}(\eta) \cdot \partial_{\eta} \Phi_{k}^{\dagger}(\eta)\right)_{+-}-\left(\Phi_{k}(\eta) \cdot \partial_{\eta} \Phi_{k}^{\dagger}(\eta)\right)_{-+}\right] .
\end{aligned}
$$

When the symmetry of the mass matrix and hence of $\Phi_{k}(\eta)$ under transposition is taken account of, one sees that there is no contribution from the second line in (4.7).

Since the Wronskian in (4.6) does not contribute to the charge (4.7), it can be written as a derivative with respect to the physical time $t$, which is related to the conformal time by $d t=a d \eta$,

$$
\begin{aligned}
q_{\phi}= & -\frac{1}{2} \frac{|\omega|}{\sqrt{\varrho^{2}-|\omega|^{2}}} \partial_{t} \int \frac{d^{3} k}{(2 \pi)^{3}}\left[\left|\Phi_{k}(\eta)\right|_{++}^{2}-\left|\Phi_{k}(\eta)\right|_{--}^{2}\right] \\
& +\frac{1}{2} \frac{\varrho}{\sqrt{\varrho^{2}-|\omega|^{2}}} \partial_{t} \int \frac{d^{3} k}{(2 \pi)^{3}}\left[\left|\Phi_{k}(\eta)\right|_{+-}^{2}-\left|\Phi_{k}(\eta)\right|_{-+}^{2}\right] .
\end{aligned}
$$


The above integrals are well known from the correlator i $\Delta\left(x, x^{\prime}\right)$ for the scalar field, which can be represented in terms of the mode sum

$$
\mathrm{i} \Delta\left(x, x^{\prime}\right)=\left\langle 0\left|\Phi\left(x^{\prime}\right) \Phi^{\dagger}(x)\right| 0\right\rangle=\int \frac{d^{3} k}{(2 \pi)^{3}} \Phi_{k}(\eta) \mathrm{e}^{\mathrm{i} \mathbf{k} \cdot \mathbf{x}} \Phi_{k}^{*}\left(\eta^{\prime}\right) \mathrm{e}^{-\mathrm{i} \mathbf{k} \cdot \mathbf{x}^{\prime}} .
$$

We recall that the Chernikov-Tagirov solution for this propagator in terms of hypergeometric functions is [5]

$$
\mathrm{i} \Delta\left(x, x^{\prime}\right)=\frac{\Gamma\left(\frac{3}{2}+\nu\right) \Gamma\left(\frac{3}{2}-\nu\right)}{(4 \pi)^{2}} H_{2}^{2} F_{1}\left(\frac{3}{2}+\nu, \frac{3}{2}-\nu ; 2 ; 1-\frac{y}{4}\right),
$$

and can be expanded as [6, 7]

$$
\begin{aligned}
\mathrm{i} \Delta\left(x, x^{\prime}\right)= & \frac{H^{2}}{4 \pi^{2}} \frac{1}{y}+\frac{H^{2}}{16 \pi^{2}} \sum_{n=0}^{\infty} \frac{\Gamma\left(\frac{3}{2}+\nu+n\right) \Gamma\left(\frac{3}{2}-\nu+n\right)}{\Gamma\left(\frac{1}{2}+\nu\right) \Gamma\left(\frac{1}{2}-\nu\right)}\left(\frac{y}{4}\right)^{n} \\
& \times\left[\log \frac{y}{4}+\psi\left(\frac{3}{2}+\nu+n\right)+\psi\left(\frac{3}{2}-\nu+n\right)-\psi(1+n)-\psi(2+n)\right]
\end{aligned}
$$

where

$$
y=\frac{-\left(\eta-\eta^{\prime}\right)^{2}+\mathbf{x}^{2}}{\eta \eta^{\prime}} .
$$

When furthermore expanding in $M^{2} / H^{2}$, we find

$$
\begin{aligned}
\mathrm{i} \Delta\left(x, x^{\prime}\right)= & \frac{H^{2}}{4 \pi^{2}} \frac{1}{y}+\frac{3 H^{4}}{8 \pi^{2} M^{2}}+\left(\frac{\log 2}{4 \pi^{2}}-\frac{7}{24 \pi^{2}}-\frac{\log y}{8 \pi^{2}}\right) H^{2} \\
& +\left(\frac{\log y}{16 \pi^{2}}-\frac{\log 2}{8 \pi^{2}}-\frac{1}{216 \pi^{2}}+O(y)\right) M^{2}+O\left(\frac{M^{4}}{H^{2}}\right) .
\end{aligned}
$$

Applying this to compute the charge (4.8), we remember that only the ++ and -- components lead to a non-vanishing result. The off-diagonal terms cancel due to the symmetry of $M^{2}$ and consequently of i $\Delta$. ¿From Eq. (4.6) it follows that the charge density is obtained by symmetrizing the time derivative as follows,

$$
q_{\phi}=-\left.\frac{|\omega|}{\varrho^{2}-|\omega|^{2}} \frac{1}{2}\left(\partial_{t}+\partial_{t^{\prime}}\right)\left[\left\langle 0\left|\Phi_{+}\left(x^{\prime}\right) \Phi_{+}(x)\right| 0\right\rangle-\left\langle 0\left|\Phi_{-}\left(x^{\prime}\right) \Phi_{-}(x)\right| 0\right\rangle\right]\right|_{x=x^{\prime}} .
$$

The term $\left.\left(\partial_{t}+\partial_{t^{\prime}}\right)(1 / y)\right|_{x=x^{\prime}}$ is singular, but these contributions cancel in (4.14), since the coefficient of $1 / y$ in (4.13) is independent of $M^{2}$. The leading contributions to $q_{\phi}$ therefore stem from the terms $\propto 1 / M^{2}$ and $\propto \log y M^{2}$. Noting $\left.\left(\partial_{t}+\partial_{t^{\prime}}\right) \log y\right|_{x=x^{\prime}}=H$, we find

$$
q_{\phi}=\left|\omega \mu^{2}\right| H \sin \theta_{\mathrm{CP}}\left[-\frac{3}{2 \pi^{2}} \frac{H^{2} \dot{H}}{m^{4}-|\mu|^{4}}+\frac{1}{8 \pi^{2}} \frac{1}{\varrho^{2}-|\omega|^{2}}\right],
$$

where the dot stands for a derivative with respect to $t$. The above expression is the main result of this paper. In appendix $\mathrm{A}$, we additionally present a derivation of the charge spectrum on super-Hubble (infrared) scales, while appendix B contains the corresponding sub-Hubble (ultraviolet) expansion. 
Let us now summarize the assumptions and approximations we have used to derive this expression for the scalar charge $q_{\phi}$. First, the expansion (4.13) is valid when $\left|M^{2}\right| \lesssim H^{2}$. To check the applicability of the result (4.15), $H^{2}$ has to be compared to the eigenvalues of the mass-squared matrix (3.26),

$M_{ \pm}^{2}=\frac{\varrho}{\varrho^{2}-|\omega|^{2}}\left[m^{2}-\frac{\left|\omega \mu^{2}\right|}{\varrho} \cos \theta_{\mathrm{CP}} \pm \sqrt{|\mu|^{4}\left(1-\frac{|\omega|^{2}}{\varrho^{2}}\right)+m^{4} \frac{|\omega|^{2}}{\varrho^{2}}-2 \frac{\left|\omega \mu^{2}\right|}{\varrho^{2}} m^{2} \cos \theta_{\mathrm{CP}}}\right]$.

Second, in order to have approximate charge conservation after inflation until the decay of $\phi$, relation (2.15) needs to hold. Taking account of these, we note that a resonant $\mathrm{CP}$ violation by setting $m \simeq|\mu|$ or $\varrho \simeq|\omega|$ cannot be achieved and that the condition $|M|^{2} \lesssim H^{2}$, for (4.16) to be valid, requires

$$
m / \sqrt{\varrho} \lesssim H
$$

While the latter source in (4.15) is fully de Sitter invariant, the former source $(\propto \dot{H})$ is obtained based on a heuristic reasoning, according to which the quasi de Sitter result can be obtained by taking the appropriate time derivative of the Hubble parameter in the de Sitter invariant propagator. This conjecture should be tested by performing the corresponding calculations with the scalar propagator constructed for quasi de Sitter spaces [3].

\section{Decay into baryons}

The above discussion shows that inflationary particle production can directly result in the generation of a charge asymmetry. We now present a possible model where this mechanism leads to a non-zero number of Standard Model baryons.

Let us assume that the neutrino masses are generated by the see-saw mechanism and that therefore a scalar condensate of $\mathrm{VEV} X$, which spontaneously breaks $\mathrm{U}(1)_{B-L}$, is present. The particle excitations associated with $X$ are taken to be ultraheavy of order of the Grand Unified scale, and we do not consider them here. We give the same $\mathrm{U}(1)_{B-L}$ charge to $\phi$ as we give to $X$. Then, the $C P$-violating parameters in the lagrangian (2.1) can be of the origin

$$
\omega=h_{\omega} X^{* 2}, \quad \mu^{2}=h_{\mu} X^{* 2} .
$$

While $h_{\mu}$ is a dimensionless parameter, $h_{\omega}$ is of mass dimension -2 , and therefore the $\omega$-term originates from a non-renormalizable operator. While there is no reason for not allowing such a term at tree-level, it will generically also be induced by radiative corrections, provided there are other operators that violate $C P$. For example, let us assume that there is an input scale $\Lambda \approx M_{\mathrm{GUT}}$, at which the $\omega$-term is absent, and let us add the operators

$$
\lambda X \phi^{* 2} \phi+\lambda^{*} X^{*} \phi^{2} \phi^{*}
$$

which give rise to cubic interactions to the lagrangian (2.1). If we assume for simplicity that $\mu=0$ and $\varrho=1$ at the scale $\Lambda$, the self-energy acting on the vector $\left(\begin{array}{ll}\phi & \phi^{*}\end{array}\right)^{T}$ reads

$$
\mathrm{i} \Pi(p)=-\frac{\mathrm{i}}{8 \pi^{2}}\left(\begin{array}{cc}
(\lambda X)^{2}+(\lambda X)^{* 2} & |\lambda X|^{2} \\
|\lambda X|^{2} & (\lambda X)^{2}+(\lambda X)^{* 2}
\end{array}\right)\left(\log \frac{\Lambda^{2}}{m^{2}}+\frac{p^{2}}{6 m^{2}}\right),
$$


where we have expanded in $p^{2}<4 m^{2}$ and $m^{2}<\Lambda$. Clearly, $\Pi\left(p^{2}\right)$ induces a radiative correction of the type of a $\mu$-like mass term and $\partial \Pi\left(p^{2}\right) / \partial p^{2}$ an $\omega$-like kinetic term.

However the CP-violating terms are generated, for an efficient charge production, we have to require $\left|M^{2}\right| \lesssim H^{2}$, corresponding to some parametric tuning. We note that for supersymmetric flat directions, mass terms of Hubble scale appear naturally from supergravity in the presence of non-vanishing $F$-terms [8]. In addition, Hubble scale mass terms are generated due the SUSY breaking in the expanding background [9].

We now need to specify how the field $\phi$ couples to Standard Model quarks and leptons. In order to allow for a decay through a tree-level, renormalizable operator, we postulate a fermion $\widetilde{H}$, that has the same gauge quantum numbers as the Standard Model Higgs field $H$. Assuming that the $B-L$ charge of $\phi$ is now one, the decay can be allowed by the renormalizable coupling

$$
\phi L \widetilde{H}+\text { h.c. }
$$

Of course, the most obvious example for such a theory is the Minimal Supersymmetric Standard Model supplemented by a right-handed neutrino, where $\phi$ should be identified with the right-handed sneutrino. This is particularly attractive, since the flatness of the potential $\phi$ arises naturally and is protected against radiative corrections by supersymmetry. It should be also noted that for a typical scale of inflation $H \sim 10^{13} \mathrm{GeV}$, the condition $|M| \lesssim H$ is usually satisfied at least by one of the heavy Majorana neutrinos [10].

\section{Baryon number of the Universe}

Now, we estimate the baryon asymmetry which results from the superhorizon fluctuations. The charge density (4.15) has two contributions: first, a term which does not vanish when $\dot{H}=0$, which we call contribution A, and second from the term $\propto \dot{H}$, which would vanish in an exact de Sitter background and which we denote by contribution B. In this section, we calculate the BAU resulting from both of these terms. The charge and baryon asymmetries from the particular contributions are called $q_{\phi}^{\mathrm{A}, \mathrm{B}}$ and $n_{B}^{\mathrm{A}, \mathrm{B}}$, and $q_{\phi}=q_{\phi}^{\mathrm{A}}+q_{\phi}^{\mathrm{B}}$ as well as $n_{B}=n_{B}^{\mathrm{A}}+n_{B}^{\mathrm{B}}$.

In order to calculate $n_{B} / s$, we assume that inflation is followed by a matter-dominated epoch after which the Universe reheats at a temperature $T_{\mathrm{R}}$ and the entropy density $s$ is produced. Let $a_{\mathrm{end}}$ denote the scale factor at the end of inflation and $a_{\mathrm{R}}$ the scale factor at reheating. We denote the charge density at reheating by $q_{\phi}^{\mathrm{R}}$, and find for the conserved charge-to-entropy ratio

$$
\frac{q_{\phi}^{\mathrm{R}}}{s}=\left(\frac{a_{\mathrm{end}}}{a_{\mathrm{R}}}\right)^{3} \frac{q_{\phi}}{s}=\frac{3}{4} \frac{T_{\mathrm{R}}}{V} q_{\phi} .
$$

When assuming instant reheating, either by fast perturbative decays or preheating of the inflaton, one gets the least dilution of the initially produced charge and

$$
T_{\mathrm{R}}=\left(\frac{30 \mathrm{~V}}{\pi^{2} g_{*}}\right)^{1 / 4}
$$

or $T_{\mathrm{R}} / V^{1 / 4}=0.41$, where $g_{*}$ is the number of relativistic degrees of freedom, and we take here the Standard Model value $g_{*}=106.75$. 
We assume that one unit of $q_{\phi}$ corresponds to $q_{B-L}$ units of $(B-L)$-asymmetry, such that $n_{B-L}=q_{B-L} q_{\phi}^{\mathrm{R}}$, and we note that sphaleron transitions convert the $(B-L)$-asymmetry into a baryon number of $n_{B}=28 / 79 n_{B-L}$. Therefore, the BAU is given by

$$
\frac{n_{B}}{s}=\frac{21}{79} \frac{T_{\mathrm{R}}}{V} q_{B-L} q_{\phi},
$$

which is to be compared to the observed value [12]

$$
\frac{n_{B}}{s} \simeq 8.7 \times 10^{-11}
$$

Let us first apply formula (6.3) to contribution A to the charge asymmetry (4.15),

$$
q_{\phi}^{\mathrm{A}}=\left|\omega \mu^{2}\right| H \sin \theta_{\mathrm{CP}} \frac{1}{8 \pi^{2}} \frac{1}{\varrho^{2}-|\omega|^{2}} .
$$

Taking account of the relations (2.12) and (4.17), it is convenient to express this as $q_{\phi}^{\mathrm{A}}=$ $\tau H^{3}$, where $\tau \lesssim 1 / 80$. We find from (6.3)

$$
\frac{n_{B}^{\mathrm{A}}}{s}=\frac{112}{79} \sqrt{\frac{2 \pi^{3}}{3}} \frac{T_{\mathrm{R}}}{V^{1 / 4}}\left(\frac{V^{1 / 4}}{m_{\mathrm{Pl}}}\right)^{3} q_{B-L} \tau=1.5 \times 10^{-9} \frac{T_{\mathrm{R}}}{0.41 V^{1 / 4}}\left(\frac{V^{1 / 4}}{10^{16} \mathrm{GeV}}\right)^{3} q_{B-L} \tau .
$$

Using the observed value of the BAU (6.4), taking for definiteness $q_{B-L}=1$ and optimistically assuming instant reheating (6.2), we find

$$
V^{1 / 4} \approx 3.9 \times 10^{15} \mathrm{GeV} \tau^{-1 / 3}
$$

We see that the baryon asymmetry depends on the magnitude of the scalar potential during inflation. Within slow-roll inflation (see e.g. [11]), it is related to the tensor-to-scalar ratio $r$ by

$$
V \approx\left(3.3 \times 10^{16} \mathrm{GeV}\right)^{4} r
$$

The present upper bound from WMAP+SDSS, assuming no running of $n_{s}$, is $r<0.30$ at $95 \%$ confidence level [12], which translates to $V^{1 / 4}<2.4 \times 10^{16} \mathrm{GeV}$. Meeting this bound requires $\tau>4.3 \times 10^{-3}$.

¿From these numbers, it is clear that contribution A only yields a sufficient abundance for large field models of inflation, where $V^{1 / 4}$ is around $10^{16} \mathrm{GeV}$. This bound is met by standard chaotic inflation with a quadratic potential, where $V^{1 / 4} \approx 2.0 \times 10^{16} \mathrm{GeV}$ at horizon exit and $V^{1 / 4} \approx 0.5 \times 10^{16} \mathrm{GeV}$ at the end of inflation. This means that contribution $\mathrm{A}$ as a source for the BAU can be ruled out by future CMB observations, if these constrain $r<$ $7 \times 10^{-2}$, when assuming that $\tau<1 / 80$. On the other hand, when assuming simple chaotic inflation, this source gives an intriguing explanation for the magnitude of the observed BAU by relating it to the scale of inflation.

Let us now turn to the contribution B to the charge (4.15),

$$
q_{\phi}^{\mathrm{B}}=-\left|\omega \mu^{2}\right| H \sin \theta_{\mathrm{CP}} \frac{3}{2 \pi^{2}} \frac{H^{2} \dot{H}}{m^{4}-|\mu|^{4}} .
$$


The time-derivative of the Hubble rate is linked to the amplitude of scalar density perturbations $\sqrt{P_{\mathcal{R}}}$ as

$$
P_{\mathcal{R}}=\frac{2^{7} \pi}{3 m_{\mathrm{Pl}}^{6}} \frac{V^{3}}{V^{\prime 2}}=-\frac{H^{4}}{\pi \dot{H} m_{\mathrm{Pl}}^{2}},
$$

where we have used the slow-roll approximation $3 H \dot{\phi}=-d V / d \phi$. The observed value at the scale $0.002 \mathrm{Mpc}^{-1}$ is $\sqrt{P_{\mathcal{R}}}=4.86 \times 10^{-5}$. Introducing

$$
\xi=\frac{\left|\omega \mu^{2}\right| H^{2}}{m^{4}-\left|\mu^{4}\right|} \sin \theta_{\mathrm{CP}},
$$

and using (6.1), we find 1

$$
\begin{aligned}
\frac{n_{B}^{\mathrm{B}}}{s} & =\frac{28}{79} q_{B-L} \frac{2^{9 / 2}}{\sqrt{3 \pi}}\left(\frac{V^{1 / 4}}{m_{\mathrm{Pl}}}\right)^{7} \frac{T_{\mathrm{R}}}{V^{1 / 4}} \frac{\xi}{P_{\mathcal{R}}} \\
& =1.2 \times 10^{-13} \frac{T_{\mathrm{R}}}{0.41 V^{1 / 4}}\left(\frac{V^{1 / 4}}{10^{16} \mathrm{GeV}}\right)^{7} q_{B-L} \xi .
\end{aligned}
$$

In this case the relations (2.12) and (4.17), which ensure the conservation of $q_{\phi}$ after inflation and the validity of our expansions, do not prevent $\xi$ from being arbitrarily large. However, it takes time until the full charge asymmetry has built up. For a light scalar field, it is a reasonable assumption that the term $S_{1}=3 H^{4} /\left(8 \pi^{2} M^{2}\right)$ in the propagator (4.13) gets replaced by $S_{2}=H^{2} N_{\mathrm{e}} /\left(4 \pi^{2}\right)$, where $N_{\mathrm{e}}=H t$ is the number of e-folds that have been elapsed since the start of inflation. When $S_{2} \simeq\left|S_{1}\right|$, the charge production is saturated and above results (6.9) and (6.12) for $q_{\phi}^{\mathrm{B}}$ and $n_{B}^{\mathrm{B}}$ apply. As long as $S_{2} \lesssim S_{1}$, one should replace $H^{2} /\left(m^{4}-\left|\mu^{4}\right|\right) \rightarrow 2 N_{\mathrm{e}} /\left(3 m^{2}\right)$ in expression (6.11) for $\xi$, recalling relation (2.12). For values of $N_{\mathrm{e}} \gtrsim 60$, just above the minimum number of e-folds required for a solution of the horizon problem, contribution $\mathrm{B}$ is therefore also a viable source for baryogenesis, provided $V^{1 / 4}$ takes large values of order $10^{16} \mathrm{GeV}$. For $N_{\mathrm{e}} \gg 60$ however, the contribution B may be much larger than the de Sitter invariant contribution A, in which case the potential can be smaller and still inflation can generate a large enough baryon asymmetry to be consistent with the observed value.

\section{Conclusions}

The CP-violating amplification of vacuum fluctuations during inflation is a viable mechanism for generation of the BAU. When assuming not much more than sixty e-folds, a number of conditions needs however to be met for successful baryogenesis.

We have found that the mechanism only leads to a sufficient baryon abundance for large scales of inflation, $V^{1 / 4} \sim 10^{16} \mathrm{GeV}$. In models operating at these energy scales, the VEV of the inflaton exceeds the Planck scale [13. In the context of local supersymmetry, supergravity corrections however spoil the slow-roll conditions when the VEVs exceeds Planck scale. On the other hand, simple chaotic inflation with a quadratic potential may still be

\footnotetext{
${ }^{1}$ Note that in this estimate we have (optimistically) assumed that the relevant $\dot{H}$ in Eq. (4.15) corresponds to the $\dot{H}$ at the time of the first Hubble crossing for the observed modes of cosmological perturbations, for which $N \simeq 50$. While this may be the case, it is by no means guaranteed.
} 
regarded as the simplest possible implementation of inflation and also deserves interest as it predicts tensor perturbations, which are observable by near-future CMB observations. We have also seen that a sufficient asymmetry requires high reheat temperatures, of the same order as $V^{1 / 4}$. This again clashes with local supersymmetry, as it would lead to a cosmological unacceptable overproduction of gravitinos.

Furthermore, the scalar field needs to decay quickly into fermions before the asymmetry $q_{\phi}$ gets altered in the post-inflationary era. Therefore, we have to assume that the CPviolating terms are smaller than the CP-conserving ones, as expressed by relation (2.12). This also means that a large Yukawa coupling $f$ is favored. We believe that a more accurate study of the evolution of the charge asymmetry after inflation is possible by matching the inflationary solutions for the scalar modes to those in radiation or matter era and will perform this in a future publication.

It is also interesting to speculate about eternal inflation [14, 15]. In the simplest case, the inflaton field is located on a local maximum of a scalar potential. While it classically tends to roll down the potential, quantum diffusion countervails such that most domains keep on inflating. Therefore, the number of e-folds can be huge and potentially a large charge asymmetry can accumulate. It should however be noted that while the inflaton is situated at the top of the potential, $\langle\dot{H}\rangle=0$, and hence $q_{\phi}^{\mathrm{B}}=0$ as in (6.9). However the squared expectation value $\left\langle q_{\phi}^{2}\right\rangle$ does not need to vanish, and it should be investigated whether it may sufficiently source baryogenesis.

In conclusion, it is not true in general that inflation dilutes any initial pre-inflationary charge asymmetry. Indeed, inflation can be used to generate a nonvanishing baryon asymmetry of the Universe. If it happened at a high energy scale, the scale of inflation will be revealed in the near future by the observation of tensor modes in the Cosmic Microwave Background. In this case the amplified charged quantum fluctuations can source a baryon asymmetry of the observed size, even under the conservative assumption that the total number of e-folds is of the order of sixty. If, on the other hand, the scale of inflation happens to be significantly lower, a sufficient amount of baryons can still be generated, provided inflation lasted much longer than 60 e-folds.

\section{A Spectrum on superhorizon scales}

Starting from the integrand in (4.8), we define the charge spectrum as

$$
\begin{aligned}
\mathcal{P}_{q_{\phi}}(k, \eta)=\frac{k^{3}}{2 \pi^{2}} q_{\phi, k}(\eta) & =\frac{k^{3}}{2 \pi^{2}}\left\{-\frac{1}{2} \frac{|\omega|}{\sqrt{\varrho^{2}-|\omega|^{2}}} \partial_{t}\left[\left|\Phi_{k}(\eta)\right|_{++}^{2}-\left|\Phi_{k}(\eta)\right|_{--}^{2}\right]\right. \\
& \left.+\frac{1}{2} \frac{\varrho}{\sqrt{\varrho^{2}-|\omega|^{2}}} \partial_{t}\left[\left|\Phi_{k}(\eta)\right|_{+-}^{2}-\left|\Phi_{k}(\eta)\right|_{-+}^{2}\right]\right\} .
\end{aligned}
$$

If we are interested in the super-Hubble part of the charge spectrum, the following small argument expansion of the mode function (3.38) is useful

$$
\Phi_{k}(\eta) \stackrel{-k \eta \ll 1}{\longrightarrow} \frac{1}{a} \frac{\Gamma(\nu)}{\sqrt{2 \pi k}} \mathrm{e}^{i(\pi / 2)(\nu-1 / 2)}\left(-\frac{k \eta}{2}\right)^{\frac{1}{2}-\nu}+\mathcal{O}(-k \eta)^{\frac{3}{2}-\nu}, \quad\left(\left|M^{2}\right| \ll H^{2}\right),
$$


such that

$$
\begin{aligned}
\left|\Phi_{k}(\eta)\right|^{2} & \stackrel{-k \eta \ll 1}{\longrightarrow} \frac{1}{a^{2}} \frac{\Gamma^{2}(\nu)}{2 \pi k}\left(\frac{k}{2 H a}\right)^{1-2 \nu}+\mathcal{O}(-k \eta)^{2-2 \nu} \\
& =\frac{2 \Gamma^{2}(\nu)}{\pi} \frac{H^{2}}{k^{3}}\left(\frac{k}{2 H a}\right)^{3-2 \nu}+\mathcal{O}(-k \eta)^{2-2 \nu}, \quad\left(\left|M^{2}\right| \ll H^{2}\right) .
\end{aligned}
$$

When $\nu \rightarrow 3 / 2$ this reduces to

$$
\left|\Phi_{k}(\eta)\right|^{2} \stackrel{M \rightarrow 0}{\longrightarrow} \frac{H^{2}}{2 k^{3}}+\mathcal{O}(-k \eta)^{2-2 \nu}, \quad(k / a \ll H),
$$

which is the scale invariant spectrum whose time derivative vanishes. However, when $M^{2} \neq 0$, one gets a contribution from the time derivative of (A.3)

$$
\partial_{t}\left|\Phi_{k}(\eta)\right|^{2} \simeq-\frac{2 \Gamma^{2}(\nu)}{\pi} \frac{H^{3}}{k^{3}}(3-2 \nu)\left(\frac{k}{2 H a}\right)^{3-2 \nu}, \quad(k / a \ll H) .
$$

where we used $\partial_{t} a=H a$. Since

$$
\nu=\frac{3}{2}-\frac{1}{3} \frac{M^{2}}{H^{2}}-\frac{1}{27} \frac{M^{4}}{H^{4}}+\mathcal{O}\left(M^{6} / H^{6}\right),
$$

Eq. (A.5) can be expanded as

$$
\partial_{t}\left|\Phi_{k}(\eta)\right|^{2} \simeq-\frac{1}{3} \frac{M^{2} H}{k^{3}}\left[1-\frac{2}{3} \frac{M^{2}}{H^{2}}\left(\ln \left(\frac{2 H a}{k}\right)+\psi(3 / 2)-\frac{1}{6}\right)\right]+\mathcal{O}\left(\frac{M^{6}}{H^{6}}\right), \quad(k / a \ll H) .
$$

The charge spectrum (A.1) can now be evaluated on super-Hubble scales to give

$$
\mathcal{P}_{q_{\phi}}(k, \eta) \simeq \frac{H}{12 \pi^{2}} \frac{|\omega|}{\sqrt{\varrho^{2}-|\omega|^{2}}}\left[m_{+}^{2}-m_{-}^{2}\right]+\mathcal{O}\left(M^{4}\right)
$$

Note that the second line in Eq. (4.7) does not contribute to the charge at leading order in $M^{2}$. Now making use of Eqs. (3.21) 3.22) we finally get

$$
\mathcal{P}_{q_{\phi}}(k, \eta) \simeq-\frac{H}{6 \pi^{2}} \frac{|\omega|}{\varrho^{2}-|\omega|^{2}}|\mu|^{2} \sin \left(\theta_{\mathrm{CP}}\right)+\mathcal{O}\left(M^{4}\right)
$$

Several comments are now in order. Firstly, the leading order contribution (the one that is linear in $M^{2} / H^{2}$ ) to the spectrum of charge is scale invariant, precisely like the spectrum of matter fluctuations in de Sitter inflation. Furthermore, as expected, the charge production vanishes when either of the parameters $\mu, \theta_{\mathrm{CP}}$ or $\omega$ vanishes, as it should. This means that the presence of the nonstandard kinetic term in the lagrangian (2.1) is essential for this charge production mechanism.

The charge phase space density generated according to (A.9) has the important property that - just as cosmological perturbations - it is frozen-in in the sense that it does not decay with the scale factor. This is so because the rate of charge dilution due to the expansion of the Universe is compensated by the creation of charge due to the CP violation present in our model (2.1). As a result, the charge density at the end of inflation is independent on the conditions at the beginning of inflation (provided of course inflation lasts long enough) and can be completely expressed in terms of the inflationary model parameters, presenting thus an unique opportunity to relate inflationary predictions tested by Cosmic Microwave Background and Large Scale Structure measurements with baryon number generation. 


\section{B Spectrum on subhorizon scales}

Let us now consider charge production on small (sub-Hubble) scales generated by our mechanism. For this purpose, the following asymptotic form of the Hankel function is useful,

$$
\begin{aligned}
H_{\nu}^{(1)}(-k \eta) & =\sqrt{-\frac{2}{\pi k \eta}} \mathrm{e}^{-i k \eta-i(\pi / 2)(\nu+1 / 2)}\left[1-\frac{\Gamma(\nu+3 / 2)}{\Gamma(\nu-1 / 2)} \frac{i}{2 k \eta}-\frac{\Gamma(\nu+5 / 2)}{\Gamma(\nu-3 / 2)} \frac{1}{8(k \eta)^{2}}\right. \\
& \left.+\cdots+\frac{\Gamma(\nu+n+1 / 2)}{\Gamma(\nu-n+1 / 2)} \frac{i^{n}}{n !(-2 k \eta)^{n}}+\mathcal{O}\left((-k \eta)^{-(n+1)}\right)\right] .
\end{aligned}
$$

From this and Eq. (3.38) we can get the asymptotic form for the mode function squared (see 8.456 in Ref. [16])

$$
\left|\Phi_{k}(\eta)\right|^{2}=\frac{1}{a^{2}} \frac{1}{2 k}\left[1+\frac{1}{2} \frac{\Gamma(\nu+3 / 2)}{\Gamma(\nu-1 / 2)}\left(\frac{H a}{k}\right)^{2}+\frac{3}{8} \frac{\Gamma(\nu+5 / 2)}{\Gamma(\nu-3 / 2)}\left(\frac{H a}{k}\right)^{4}+\mathcal{O}\left((a H / k)^{6}\right)\right],
$$

such that

$$
\partial_{t}\left|\Phi_{k}(\eta)\right|^{2}=-\frac{1}{a^{2}} \frac{H}{k}+\frac{3}{8} a^{2} \frac{\Gamma(\nu+5 / 2)}{\Gamma(\nu-3 / 2)}\left(\frac{H}{k}\right)^{5}+\mathcal{O}\left((a H / k)^{6}\right) .
$$

This can be expanded in powers of $M^{2} / H^{2}$,

$$
\partial_{t}\left|\Phi_{k}(\eta)\right|^{2} \simeq-\frac{1}{a^{2}} \frac{H}{k}-\frac{3}{4} a^{2} \frac{H^{3} M^{2}}{k^{5}}\left[1-\frac{1}{2} \frac{M^{2}}{H^{2}}\right]+\mathcal{O}\left(M^{2}(a H / k)^{6}\right) .
$$

The first (leading) term does not contribute to the charge spectrum (A.1), such that on sub-Hubble scales the charge generation is suppressed as

$$
\mathcal{P}_{q_{\phi}}(k, \eta) \simeq \frac{3}{16 \pi^{2}} \frac{a^{2} H^{3}}{k^{2}} \frac{|\omega|}{\sqrt{\varrho^{2}-|\omega|^{2}}}\left[m_{+}^{2}-m_{-}^{2}\right] .
$$

Making use of Eq. (3.21 3.22 ) we can evaluate the charge spectrum (B.5),

$$
\mathcal{P}_{q_{\phi}}(k, \eta) \simeq-\frac{3}{8 \pi^{2}} \frac{a^{2} H^{3}}{k^{2}} \frac{|\omega|}{\varrho^{2}-|\omega|^{2}}|\mu|^{2} \sin \left(\theta_{\mathrm{CP}}\right) .
$$

An important property of this spectrum is that the charge density is ultraviolet finite. Indeed, when the charge spectrum (B.5) is integrated out, the dominant contribution comes from the modes $k \sim H a$, which is the most infrared mode for which the asymptotic expansion makes sense. We thus conclude that $\left.q_{\phi}\right|_{\mathrm{UV}} \sim|\mu|^{2} H\left(|\omega| / \varrho^{2}\right) \sin \left(\theta_{\mathrm{CP}}\right)$. This then implies that to the leading approximation, one can neglect the charge production on sub-Hubble scales in our model.

\section{References}

[1] A. D. Sakharov, "Violation of CP Invariance, c Asymmetry, and Baryon Asymmetry of the Universe," Pisma Zh. Eksp. Teor. Fiz. 5 (1967) 32 [JETP Lett. 5 (1967 SOPUA,34,392-393.1991 UFNAA,161,61-64.1991) 24]. 
[2] S. van der Post and T. Prokopec, "Baryogenesis in Brans-Dicke theory," arXiv:hep-ph/0612313.

[3] T. Janssen and T. Prokopec, in progress (2007).

[4] T. S. Bunch and P. C. W. Davies, "Quantum Field Theory In De Sitter Space: Renormalization By Point Splitting," Proc. Roy. Soc. Lond. A 360 (1978) 117.

[5] N. A. Chernikov and E. A. Tagirov, "Quantum theory of scalar fields in de Sitter space-time," Annales Poincare Phys. Theor. A 9 (1968) 109.

[6] T. Prokopec and E. Puchwein, "Photon mass generation during inflation: de Sitter invariant case," JCAP 0404 (2004) 007 [arXiv:astro-ph/0312274].

[7] B. Garbrecht and T. Prokopec, "Fermion mass generation in de Sitter space," Phys. Rev. D 73 (2006) 064036 [arXiv:gr-qc/0602011.

[8] M. Dine, L. Randall and S. D. Thomas, "Baryogenesis From Flat Directions Of The Supersymmetric Standard Model," Nucl. Phys. B 458 (1996) 291 arXiv:hep-ph/9507453.

[9] B. Garbrecht, "Radiative lifting of flat directions of the MSSM in de Sitter background," arXiv:hep-ph/0612011.

[10] W. Buchmüller, R. D. Peccei and T. Yanagida, "Leptogenesis as the origin of matter," Ann. Rev. Nucl. Part. Sci. 55 (2005) 311 [arXiv:hep-ph/0502169].

[11] D. H. Lyth and A. Riotto, "Particle physics models of inflation and the cosmological density perturbation," Phys. Rept. 314 (1999) 1 |arXiv:hep-ph/9807278|.

[12] D. N. Spergel et al. [WMAP Collaboration], "Wilkinson Microwave Anisotropy Probe (WMAP) three year results: Implications for cosmology," arXiv:astro-ph/0603449.

[13] D. H. Lyth, "What would we learn by detecting a gravitational wave signal in the cosmic microwave background anisotropy?," Phys. Rev. Lett. 78 (1997) 1861 arXiv:hep-ph/9606387.

[14] A. D. Linde, "Nonsingular Regenerating Inflationary Universe," Preprint PRINT-820554-CAMBRIDGE.

[15] A. Vilenkin, "The Birth Of Inflationary Universes," Phys. Rev. D 27 (1983) 2848.

[16] Izrail Solomonovich Gradshteyn, Iosif Moiseevich Ryzhik, Table of integrals, series, and products, 4th edition, Academic Press, New York (1965). 